\title{
Infrastructureless UWB based collision avoidance system for the safety of construction workers
}

\author{
$1^{\text {st }}$ Marianna Pittokopiti \\ Dept. of Electronic and Electrical Engineering \\ University College London \\ London, United Kingdom \\ marianna.pittokopiti.13@ucl.ac.uk
}

\author{
$2^{\text {nd }}$ Ryan Grammenos \\ Dept. of Electronic and Electrical Engineering \\ University College London \\ London, United Kingdom \\ r.grammenos@ucl.ac.uk
}

\begin{abstract}
Collisions with vehicles are one of the leading causes for fatal and non-fatal accidents at construction sites. This paper discusses the implementation of a low-cost, battery-powered Ultra-Wideband (UWB) based collision avoidance system for use in the construction industry that can detect potential collisions between workers and vehicles in real-time. The key advantage of our proposed system compared to existing solutions is that it does not require a fixed infrastructure. We also introduce an additional metric being the time to collision, beyond the standard distance measurements. Results show that the combination of UWB and linear regression provides sufficient accuracy, with a mean error of $0.75 \mathrm{~m}$ in distance measurements and less than $1 \mathrm{~s}$ error in the time to collision for relative speeds up to $2.65 \mathrm{~m} / \mathrm{s}$. This error is even smaller for higher speeds encountered in real-life scenarios.
\end{abstract}

Index Terms-Ultra-wideband, UWB, collision avoidance, distance measurement, time to collision, linear regression.

\section{INTRODUCTION}

In $2017 / 18$, the construction industry was the industry with the most work related injuries. Being struck by a moving vehicle or object was the second most common cause of fatal injuries across all industries and the third most common cause of non-fatal injuries in the construction industry [1]. The Health and Safety Executive (HSE) has reported that 7 workers die and 93 are seriously injured on average each year (in the United Kingdom), as a result of accidents involving vehicles or mobile plants on construction sites [2].

This work presents the development of a collision avoidance system targeted for the use on smart vests for construction workers. The aim is to devise a solution that reduces accidents involving vehicles in construction sites, by using audiovisual notifications to warn the workers when a vehicle or crane is approaching. Ultra-Wideband (UWB) communication is used as the main technology for measuring the distance between workers and vehicles, with a theoretical accuracy of 0.1-0.5 m [3]. The aim of this work is to achieve a distance measurement accuracy within the range $\pm 0.5-1.0 \mathrm{~m}$ and a communication range of at least $30 \mathrm{~m}$, so that the worker has sufficient time to respond after being notified of the danger. This level of accuracy is sufficient for this kind of application, since the error in the calculation of the time until the collision occurs would correspond to less than $1 \mathrm{~s}$ if we take into account vehicle speeds on sites, which are typically greater than 5 $\mathrm{m} / \mathrm{s}$. Additionally, UWB transceivers consume power in the $\mathrm{mA}$ range making them suitable for battery-powered solutions [4]. Another benefit of the proposed solution is that it does not require a fixed infrastructure, since the modules will be placed directly on workers or in vehicles.

This paper is organised as follows; Section II outlines related work while Section III discusses the system design and implementation. Results are presented in Section IV with Section V concluding this paper.

\section{RELATED WORK}

There is extensive literature reporting the use of telecommunications for worker safety related to moving vehicles at construction sites. Technologies such as global positioning system (GPS), dedicated short-range communications (DSRC) and radio frequency identification (RFID) are typically employed in systems designed to protect workers from incoming vehicles or other moving equipment [5], [6].

UWB communications have been used [3], [7], [8] to limit accidents at construction sites by identifying the distance between the worker and an imminent danger, such as approaching vehicles or other dangerous equipment. Research in UWB localisation for industrial applications [8], [9], [10] further support the use of UWB in such scenarios. Even though sufficient accuracy was achieved, existing solutions [3], [7] necessitate a fixed UWB infrastructure.

Hence, this work presents the realisation of an infrastuctureless UWB based solution that can achieve sufficient accuracy at lower costs and simpler design complexity compared to current state-of-the-art solutions. An example of a similar Arduino-based UWB positioning solution is Pozyx [11]. Yet, the price for a Pozyx anchor and tag is around $\$ 300$, which is approximately three times more expensive compared to our proposed prototype system.

\section{System Design AND IMPLEMENTATION}

UWB communication operates in the $3.1-10.6 \mathrm{GHz}$ band and occupies a very wide bandwidth $(500 \mathrm{MHz}$ in this case, but larger bandwidths are also available). This allows the spectral density to be very low, sometimes even lower than the noise coming from other electronic equipment, thus not interfering with other radio technologies. Since an UWB 
(a)
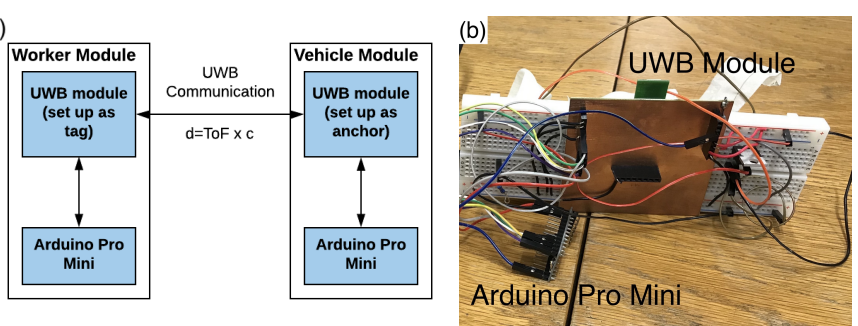

Fig. 1: (a) System diagram of the proposed solution. (b) System prototype of an UWB module configured as a tag/anchor and connected to an Arduino Pro Mini.

pulse is transmitted typically in less than one nanosecond, the reflected pulse only has a very small probability of interfering with the original pulse [4]. Depending on the configuration used, the practical accuracy varies between 0.1 and $1.0 \mathrm{~m}$, and the communication range can exceed $100 \mathrm{~m}$ (RF antenna dependent) [3], [12].

The UWB transceiver selected for this project is the DWM1000 module by DecaWave. It is supported by an opensource Arduino library on Git-Hub [13] that defines a twoway-ranging (TWR) algorithm to calculate the distance between two modules using (1). The DWM1000 module requires no RF design as the antenna and associated analog and RF components are located on the module.

In order to detect potential collisions between workers and vehicles, the distance between them needs to be measured continuously and in real-time. To measure the distance between a worker and a vehicle, the module assigned to a worker is set up as a tag while the module assigned to a vehicle is set up as an anchor, as illustrated in Fig. 1a, where each UWB module is controlled by an Arduino Pro Mini. Fig. 1b depicts a system prototype for a worker module. It is worth noting that worker and vehicle modules are identical in terms of hardware with their relevant function configured in software. Both the microcontroller and the UWB module can be powered from four $1.5 \mathrm{~V}$ AA batteries in series regulated down to $3.3 \mathrm{~V}$.

TWR can calculate the distance between two objects by determining the Time of Flight ( $\mathrm{ToF}$ ) of signals travelling between them. The ToF is calculated using the timestamps generated by a message exchange between the anchor and the tag with the distance given by:

$$
d_{M}=t_{T o F} \times c
$$

where $d_{M}$ is the measured distance between the anchor and the tag, $t_{T o F}$ is the time of flight and $c$ is the speed of light [14]. TWR has the advantage that it does not require exact synchronisation between the anchors and the tags, unlike Time Difference of Arrival (TDoA). Moreover, it does not require any fixed device infrastructure to determine the separation distances.

The communication range between two DWM1000 UWB modules is determined based on the received signal strength indicator (RSSI) and the sensitivity of the receiver. In order to establish a communication link between the two modules, the received signal strength should be greater than or equal to the sensitivity of the receiver. Equation (2) describes how the RSS might be affected by an obstruction [15]:

$$
\begin{aligned}
& P_{R}[d B m]=P_{T}[d B m]+G[d B]-L[d B] \\
& \quad-20 \log 10\left(4 \pi f_{c}\left(d_{1}+d_{2}\right) / c\right)-L_{\text {material }}[d B]
\end{aligned}
$$

where $P_{R}$ is the received signal strength, $P_{T}$ is the transmitted power, $G$ is the sum of the gains of the transmitting and receiving antennas, $L$ represents the losses in the system, $c$ is the speed of light, $f_{c}$ is the channel's center frequency in Hertz, $d_{1}$ is the free space distance from the transmitter to the material causing the obstruction, $d_{2}$ is the free space distance from the same material to the receiver, and $L_{\text {material }}$ is the loss caused by the obstructing material [15].

\section{A. Collision Avoidance Algorithm}

Assuming the distance versus time curve is a collection of linear regions, the least squares linear regression method can be used to determine the gradient of the linear regions of the distance-time curve. Equation (3) gives the gradient $g$ of a line:

$$
g=\frac{n\left(\sum x y\right)-\left(\sum y\right)\left(\sum x\right)}{n\left(\sum x^{2}\right)-\left(\sum x\right)^{2}}
$$

where $x$ and $y$ represent the $\mathrm{x}$-axis and $\mathrm{y}$-axis values respectively, and $n$ is the number of data points. The least squares function used in the collision avoidance algorithm below is based on (3). The gradient, which gives the relative speed between the two modules, can be used subsequently to calculate the time until a collision occurs between a worker and a vehicle, or to identify whether the distance between the two is decreasing by checking if the gradient is negative.

The distance between worker and vehicle is measured continuously with the TWR algorithm running on the microcontroller controlling the UWB modules and then used to compute the gradient of the distance versus time. The measured distance and the corresponding timestamp are saved in an array that can hold 10 sets of measurements. A new distance measurement is made approximately every $0.1 \mathrm{~s}(9 \mathrm{~Hz})$ and the gradient is calculated every time a new measurement is made. The distance measurement and collision avoidance algorithm are running continuously as long as the Arduino Pro Mini is powered, and there is at least one tag paired with one anchor.

Empirical evaluation showed that the distance is considered to be decreasing when the gradient is smaller than $-0.1 \mathrm{~m} / \mathrm{s}$, accounting for minor fluctuations in the distance measurements or small movements of the worker while standing in the same position. The time to collision $\left(t_{c o l}\right)$ is calculated by dividing the measured distance by the absolute value of the gradient, and an audiovisual notification is generated according to predefined $t_{c o l}$ thresholds.

The collision avoidance algorithm may be summarised as follows:

loop

while tag paired with anchor do 


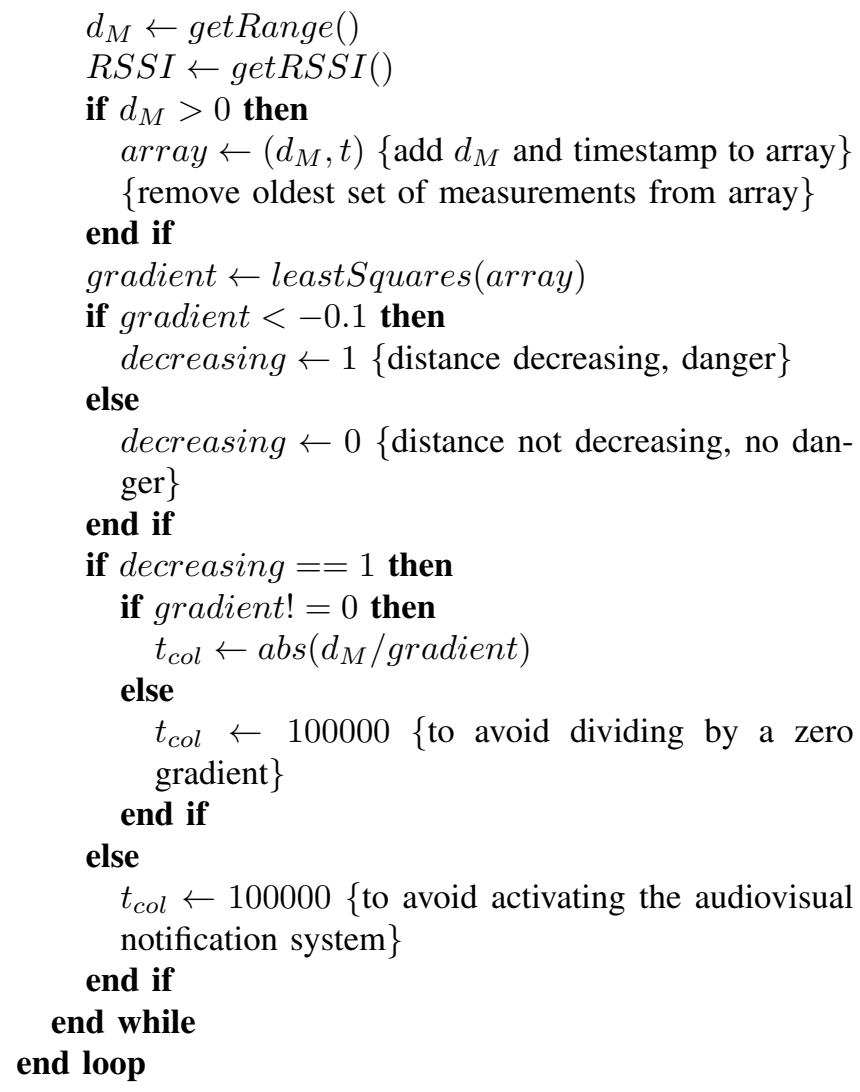

\section{B. Experimental Procedures}

In this work, one UWB module was set up as an anchor and one as a tag. The Arduino IDE was used to run the collision avoidance algorithm. The low power mode of the UWB module was used that transmits data at a rate of $110 \mathrm{kbps}$ with a pulse frequency of $16 \mathrm{MHz}$. The channel bandwidth was $500 \mathrm{MHz}$ [4]. The following experiments were performed:

1) UWB Received Signal Strength and Range Test: The received signal strength (RSS) was recorded while the distance between the two modules was increased and then plotted against the distance so as to investigate their relationship. Additionally, in order to assess the line-of-sight (LOS) range of communication outdoors, the distance between the two devices was increased continuously until no further communication could take place and then that distance was recorded.

2) UWB Module NLOS Behaviour Test: The behaviour of the UWB system under non-LOS (NLOS) conditions was tested by standing $2 \mathrm{~m}$ in front of the receiver so as to create an obstruction, while the distance between the two modules was kept constant. The received signal strength and the distance calculated by the TWR algorithm were recorded.

3) UWB Distance Measurement Accuracy: In order to assess the accuracy of the distance measurements under LOS conditions, the distance between the two modules was varied and the measured and actual distances were recorded. This experiment was conducted in a long corridor indoors where the environment is more controlled and it is less likely for people to interrupt the measurement by accident (since obstructions between the two modules would affect the recorded readings).
The two modules were placed opposite each other at the same height. Marks were placed on the floor at increments of $2 \mathrm{~m}$ away from the receiver, for a total distance of 32 $\mathrm{m}$. A distance of $32 \mathrm{~m}$ was chosen because the aim was to provide a communication range of at least $30 \mathrm{~m}$ while providing sufficient time to explore the behaviour of the collision avoidance algorithm as the distance between the two modules decreases. Two hundred measurements were recorded at each marked location and then averaged to find the mean measured distance for each mark. The error for each known distance was calculated by subtracting the actual distance from the measured one.

4) Collision Avoidance Algorithm Performance Evaluation: The collision avoidance algorithm using the distance measurements from the UWB modules was tested. This test was carried out indoors, in the same corridor as experiment (3) and the vehicle module was attached on top of a remotecontrolled toy car. The worker module (tag) was held constant while the vehicle module (anchor) was approaching (speed $<2.97 \mathrm{~m} / \mathrm{s}$ ). The initial distance between the two modules was approximately $28.6 \mathrm{~m}$. The distance between the two modules, the calculated gradient, the time to collision, as well as the elapsed time, were recorded as the vehicle module was approaching the worker module. The recordings were then processed offline to assess the performance and accuracy of the algorithm. Since the mean error of the distance measurements is known, the error in the time to collision can be calculated.

Experiment (4) was repeated three more times. The initial distance measured using UWB and the distance when the collision was first detected were recorded in order to assess whether the possible collision was detected and whether the collision was detected with sufficient distance between the worker and vehicle modules.

Experiment (4) was also repeated with the vehicle module moving with walking speeds (speed $<1.50 \mathrm{~m} / \mathrm{s}$ ). The initial distance between the modules was in the range of 20-25 $\mathrm{m}$ and the vehicle module was moving in a straight line along the corridor. These distances were selected because the possible collision must be detected at least $20 \mathrm{~m}$ away from the worker, in order to give them enough time to escape the imminent danger. The vehicle module was stopped at different known distances from the worker module. Given that the actual distance is known, the error in the distance measurement can be calculated and accounted for.

\section{RESUlts AND ANALYSIS}

1) UWB Received Signal Strength and Range: The RSS against the distance was recorded while the distance between the tag and the anchor was increased until the communication link failed (under LOS conditions). The results are plotted in Fig. 2. It can be observed that the communication link failed when the RSS was approximately $-107 \mathrm{dBm}$. The communication range at that point was $91.68 \mathrm{~m}$, which is more than sufficient for the application under consideration.

2) UWB module NLOS behaviour: When an object obstructs the LOS path between the transmitter and the receiver, 


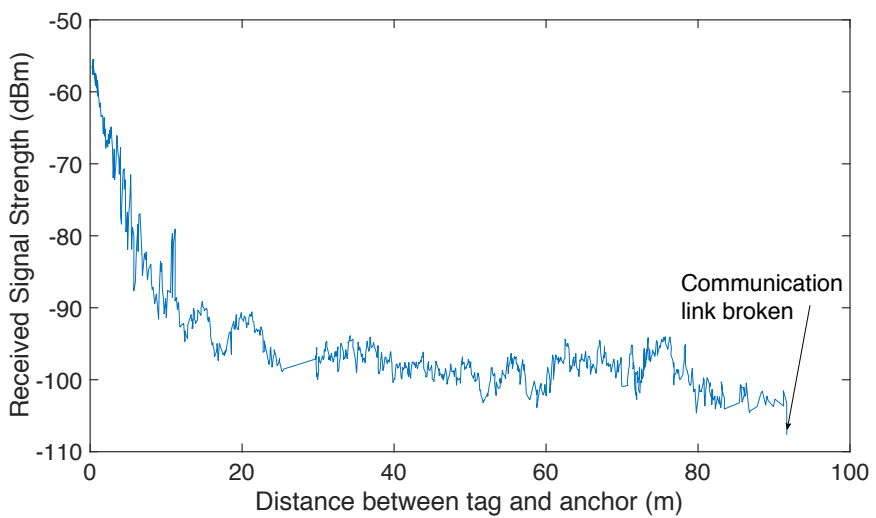

Fig. 2: Variation of the Received Signal Strength with increasing distance until the communication link is broken.

the signal is attenuated, yet as long as its power is above the sensitivity of the receiver, it can still be sensed and the communication link is maintained. Fig. 3 shows that when an object obstructs the LOS path between the transmitter and the receiver (middle section of the plot, sample index 80-140), the measured distance $d_{M}$ increases, even though the actual distance between the transmitter and the receiver remains constant. This, in turn, increases the error in the distance measurements. At the same time, the RSSI decreases accordingly due to the attenuation caused by the obstruction.

These results confirm that the UWB system performs better under LOS conditions. Obstructions will reduce the accuracy of the system, however, in such cases, there is unlikely any danger to begin with, since an obstruction already lies in the direct path between the worker and the vehicle.

3) UWB Distance Measurement Accuracy: Fig. 4 shows that the mean error in distance measurements is $0.75 \mathrm{~m}$. The results of this experiment illustrate that for the open-source library (TWR algorithm) and the UWB module used, the error in the distance measurements is always additive, that is, the

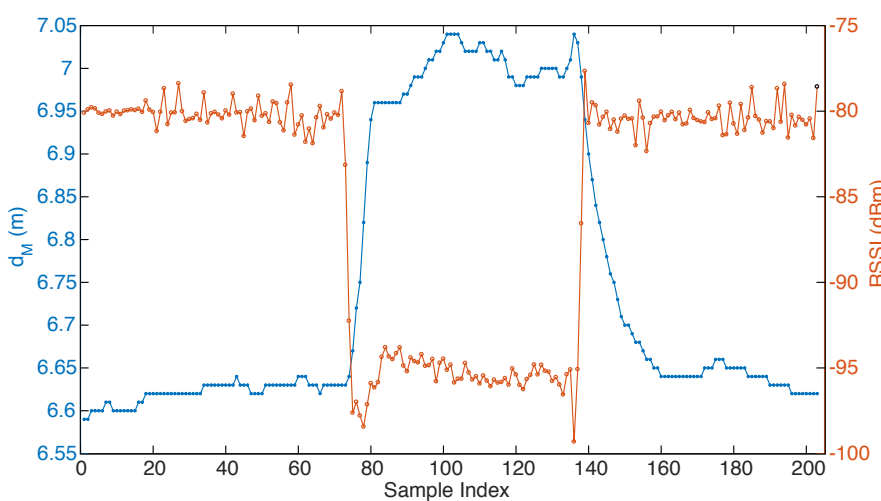

Fig. 3: Effect of a human body obstructing the UWB LOS communication path between anchor and tag on (a) the distance measurement accuracy (left y-axis), and (b) the received signal strength indicator (right $\mathrm{y}$-axis).

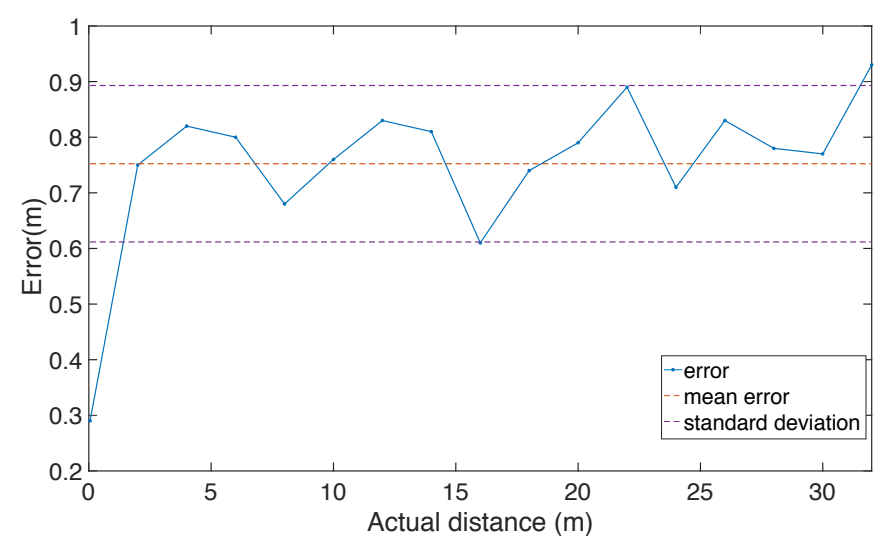

Fig. 4: Measured distance error against actual distance.

error always causes an overestimation of the distance. The error in the distance measurements is within the $\pm 0.5-1.0 \mathrm{~m}$ range specification defined at the outset. If we assume that the worker is standing still, the vehicle is moving with a speed of $5 \mathrm{~m} / \mathrm{s}$ towards the worker and that the error is $1 \mathrm{~m}$ (which is more that the amount of error actually observed), that would translate into an error of $0.2 \mathrm{~s}$ when calculating the time to collision. For higher speeds, for example $15 \mathrm{~m} / \mathrm{s}$, the error in the time to collision would be even smaller.

4) Evaluation of the collision avoidance algorithm: The performance of the collision avoidance algorithm was found to be dependent on the gradient of the measured distance-time curve and the value of the error in the distance measurements. The gradient $g$ is given by:

$$
g=\frac{y_{2}-y_{1}}{x_{2}-x_{1}}
$$

where $y_{1}, y_{2}$ are successive distance measurements, and $x_{1}$, $x_{2}$ are successive timestamps. Since the error in distance measurements is always additive (as shown in Section IV-3), and assuming the error in successive distance measurements will be almost the same, only a negligible error will remain in the gradient after subtracting $y_{1}$ from $y_{2}$. Hence, it can be assumed that the error in the calculated gradient due to the error in distance measurements is negligible. The measured distance between anchor and tag is given by:

$$
d_{M}=d_{A}+\epsilon
$$

where $d_{A}$ is the actual distance between anchor and tag and $\epsilon$ is the error between the actual and measured distances. The time to collision may therefore be expressed as:

$$
t_{c o l}=\frac{d_{M}}{|g|}=\frac{d_{A}+\epsilon}{|g|}
$$

The second term of (6) gives the error $e_{\text {calc }}$ in the calculation of the time to collision, expressed as:

$$
e_{\text {calc }}=\frac{\epsilon}{|g|}
$$

Equation (7) proves that as the relative speed of the worker 

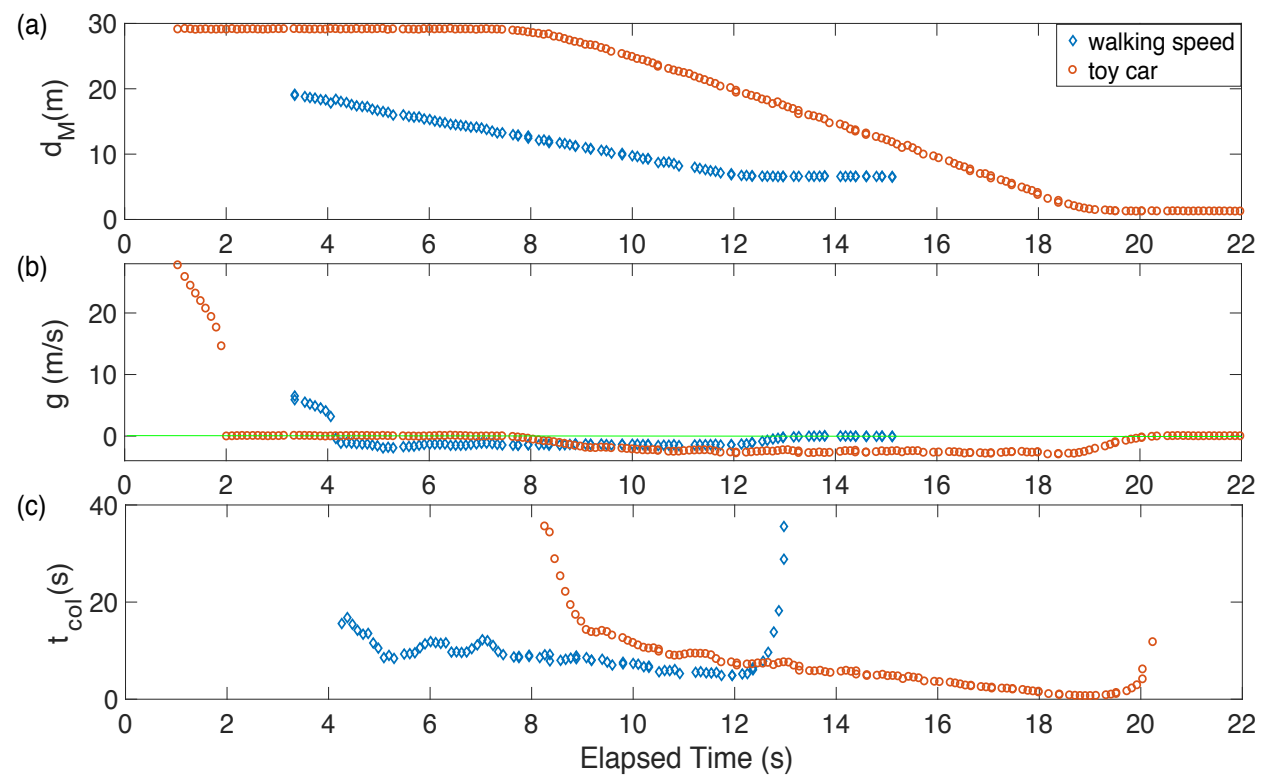

Fig. 5: a) Measured distance, b) gradient of distance against time, and c) time to collision, against elapsed time for two test cases, remote-controlled toy car (red circles) and walking speed (blue diamonds).

and vehicle increases, the error in the calculated time to collision decreases, since the error is inversely proportional to the gradient.

Fig. 5a illustrates how the distance measured using the UWB modules varies with elapsed time for two cases; toy car speeds $(<2.97 \mathrm{~m} / \mathrm{s})$ and walking speeds $(<1.50 \mathrm{~m} / \mathrm{s})$. As expected, the gradient is steeper for the measurements acquired using the toy car.

Concentrating on the results for the "toy car" case (red circles):

During the first second of the measurement $(0-1 \mathrm{~s})$, the microcontroller is busy executing initialisation processes and hence no data is being acquired. Fig. 5a shows that the toy car was stationary at the start of the measurement (1-8 s), which is the reason that the $t_{c o l}$ results are only present for an elapsed time of $8 \mathrm{~s}$ onwards.

Fig. 5b shows that at the start of the data acquisition $(1-2 \mathrm{~s})$, the values for the gradient are quite large $(>10$ $\mathrm{m} / \mathrm{s}$ ). This is due to the initialisation of the microcontroller code where the measurement array is populated with zeros. Every time a new measurement is added, the linear regression algorithm only sees an increase in the distance. Since the measurement array can hold only 10 sets of distance and timestamp measurements, and a new measurement is added approximately every $0.1 \mathrm{~s}$, the algorithm needs about $1 \mathrm{~s}$ to realise whether the distance is increasing, decreasing or remains constant. The absolute value of the gradient gives the relative speed of the worker and the vehicle, as discussed in Section III-A.

At $18.5 \mathrm{~s}$ (Fig. 5b), the gradient increases gradually up to zero because the distance between the two modules at that point is constant. This increase is a result of the algorithm using the last 10 distance-time measurements to calculate the gradient. This means that for the algorithm to calculate a gradient equal to, or approximately equal to, zero, ten consecutive equidistance measurements need to be saved in the array.

Finally, Fig. 5c depicts the time to collision, as explained in Section III-B4. When the gradient is equal to zero or positive, as it is at the start and the end of the measurement, the variable holding the time to collision is assigned the value 100000 in the code since at that time there is no risk of a collision. This also prevents dividing the distance by zero. The data points related to this effect are not shown on the plot since that value is out of the y-axis boundaries of the plot. When the gradient is negative, the value of $t_{c o l}$ is calculated as explained in Section III-A.

The error in the calculation of the time to collision, $e_{\text {calc }}$, depends on the error of the TWR algorithm measuring the distance between the UWB anchor and tag, as well as the relative speed of the two modules. Consequently, the measurements for the toy car in Fig. 5 give an error of $0.28 \mathrm{~s}$ in the calculation of the time to collision. This is also indicated in Table I for Trial A. In real-life scenarios where the relative speed will be greater than $5 \mathrm{~m} / \mathrm{s}$, this time to collision error will be much smaller.

The curves with the blue diamonds in Fig. 5 depict the results when the same experiment was carried out but with the anchor moving at walking speeds $(<1.50 \mathrm{~m} / \mathrm{s})$. It is worth noting that at the start of this measurement, the microcontroller took longer to reset and reinitialise and hence the data acquisition only started after $3 \mathrm{~s}$. It is evident that the plots follow the same trend as those illustrated for the toy car scenario (red circles). Yet, the higher speed achieved with the toy car 
TABLE I: Collision avoidance results and corresponding error in the time to collision $e_{\text {calc }}$ for different trials.

\begin{tabular}{|c|c|c|c|c|c|}
\hline Trial & $d_{M} \mathbf{( m )}$ & $d_{A} \mathbf{( m )}$ & $g(\mathbf{m} / \mathbf{s})$ & $t_{\text {col }}(\mathbf{s})$ & $e_{\text {calc }}(\mathbf{s})$ \\
\hline A-Toy car & 3.76 & 3.01 & -2.65 & 1.42 & 0.28 \\
\hline B-Walking & 6.73 & 6.00 & -1.28 & 5.26 & 0.57 \\
\hline C-Walking & 8.67 & 8.00 & -1.04 & 8.33 & 0.64 \\
\hline D-Walking & 6.66 & 6.00 & -0.90 & 7.36 & 0.73 \\
\hline E-Walking & 2.66 & 2.00 & -0.84 & 3.16 & 0.79 \\
\hline F-Walking & 4.70 & 4.00 & -0.83 & 5.66 & 0.84 \\
\hline
\end{tabular}

TABLE II: Detection accuracy of UWB algorithm for different trials with the anchor module attached on a toy car.

\begin{tabular}{|c|c|c|c|}
\hline Trial & Detected & $\begin{array}{c}d_{M} \text { when collision } \\
\text { is first detected }(\mathbf{m})\end{array}$ & $\begin{array}{c}\text { Initial } d_{M} \text { measured } \\
\text { using UWB } \mathbf{~ ( m )}\end{array}$ \\
\hline 1 & Yes & 28.92 & 29.09 \\
\hline 2 & Yes & 28.62 & 29.07 \\
\hline 3 & Yes & 28.39 & 28.62 \\
\hline
\end{tabular}

results in a steeper gradient and hence the error in the time to collision is much smaller. This result can be better understood by comparing Trials A (toy car speed) and B (walking speed) in Table I, where it can be observed that when the gradient is almost doubled, the error is approximately halved.

Table I presents the calculated error in the time to collision for different trials, calculated using (7), at specific distances throughout each measurement. It is evident that for relative speeds up to $2.65 \mathrm{~m} / \mathrm{s}$ (represented by the absolute value of the gradient), the error $e_{\text {calc }}$ is less than $1 \mathrm{~s}$. Furthermore, this error decreases as the relative speed of the worker and the vehicle increases, which suggests promising results for practical deployments.

Table II indicates that for three tests conducted using the toy car, a possible collision was always detected at sufficient distance away from the worker, in this case more than 20 $\mathrm{m}$ away. The three tests reported a $100 \%$ accuracy in the detection of a decrease in the distance between the two UWB modules. These results imply that the worker will be notified in good time to avoid any imminent danger.

To conclude, even though the target environment for this work was an outdoor construction site, the proposed solution would also function in indoor environments such as warehouses, thanks to the capability of UWB communications to operate effectively both in indoor and outdoor environments.

\section{CONCLUSION}

This paper presented the development of a real-time collision avoidance system targeted for use on construction sites. Experimental results show that the designed solution was able to measure distance and determine potential collisions with sufficient accuracy to protect workers.

The UWB communication link was found to be robust up to $91 \mathrm{~m}$ under LOS conditions, allowing the system to detect a danger from far away and hence giving workers sufficient time to respond to danger. The error of the collision avoidance algorithm developed was found to be less than $1 \mathrm{~s}$ for relative speeds up to $2.65 \mathrm{~m} / \mathrm{s}$ and it was proven that as the relative speed of the worker and vehicle increases, the error (in seconds) decreases.

All the above suggest that the combination of UWB communications with a linear regression algorithm is a very good candidate for collision avoidance applications, offering sufficient accuracy at a low cost with the added advantage of not requiring a fixed infrastructure.

Future work will look at incorporating this system on a smart vest that will notify construction workers when they are in danger from an approaching vehicle or crane, scaling up the system to support multiple anchors and tags, as well as carrying out further tests on construction sites.

\section{ACKNOWLEDGMENT}

The authors would like to acknowledge and thank Arman Assylkhan whose final year project inspired the work presented in this paper.

\section{REFERENCES}

[1] Health and Safety Executive UK. Workplace statistics in Great Britain 2018. [Online]. Available: http://www.hse.gov.uk/statistics

[2] —. Traffic management on site. [Online]. Available: http://www.hse. gov.uk/construction/safetytopics/vehiclestrafficmanagement.htm

[3] A. Carbonari, A. Giretti, and B. Naticchia, "A proactive system for real-time safety management in construction sites," Automation in Construction, vol. 20, no. 6, pp. 686 - 698, 2011, selected papers from the 26th ISARC 2009. [Online]. Available: http: //www.sciencedirect.com/science/article/pii/S0926580511000756

[4] Decawave. DWM1000 IEEE 802.15.4-2011 UWB Transceiver Module Datasheet. [Online]. Available: https://www.decawave.com/sites/default/ files/resources/DWM1000-Datasheet-V1.6.pdf

[5] J. Forsyth, T. Martin, and D. Bowman, "Feasibility of gps-based warning system for roadside workers," in 2014 International Conference on Connected Vehicles and Expo (ICCVE), Nov 2014, pp. 1045-1051.

[6] G. L. Amicucci and F. Fiamingo, "Usage of rfid in safety applications," in 2017 IEEE International Conference on Environment and Electrical Engineering and 2017 IEEE Industrial and Commercial Power Systems Europe (EEEIC / I CPS Europe), June 2017, pp. 1-6.

[7] D. J.Teizer and M. Sofer, "Rapid automated monitoring of construction site activities using ultra-wide band," 092007.

[8] H. Yang and W. Wu, "Uwb-assisted real-time localization in wireless sensor networks," in IECON 2013 - 39th Annual Conference of the IEEE Industrial Electronics Society, Nov 2013, pp. 4500-4505.

[9] B. Silva, Z. Pang, J. kerberg, J. Neander, and G. Hancke, "Experimental study of uwb-based high precision localization for industrial applications," in 2014 IEEE International Conference on Ultra-WideBand (ICUWB), Sep. 2014, pp. 280-285.

[10] — "Positioning infrastructure for industrial automation systems based on uwb wireless communication," in IECON 2014 - 40th Annual Conference of the IEEE Industrial Electronics Society, Oct 2014, pp. 3919-3925.

[11] Pozyx. UWB Accurate Positioning. [Online]. Available: https://www. pozyx.io/

[12] H. Li, G. Chan, J. K. W. Wong, and M. Skitmore, "Realtime locating systems applications in construction," Automation in Construction, vol. 63, pp. 37 - 47, 2016. [Online]. Available: http://www.sciencedirect.com/science/article/pii/S0926580515002411

[13] DW1000 Library GitHub Contributors. A library that offers functionality to use Decawave's DW1000 chips/modules with Arduino. [Online]. Available: https://github.com/thotro/arduino-dw1000

[14] Decawave. APS013: The implementation of two-way ranging with the DW1000. [Online]. Available: https://www.decawave.com/sites/default/ files/aps013_dw1000_and_two_way_ranging_v2.2.pdf

[15] - APS006: Channel Effects on Communications Range and Time Stamp Accuracy in DW1000 based systems. [Online]. Available: https://www.decawave.com/sites/default/files/aps006 \_channel $\backslash$ _ effects $\backslash \_$on $\backslash$ range $\backslash \_$accuracy.pdf 